\title{
To Evaluate Bacterial Contamination of Surgical Handpiece Used in Minor Oral Surgical Procedures in Dentistry
}

\author{
Swetha Murali ${ }^{1}$, Madhulaxmi M², Muthusekar M.R. ${ }^{3}$ \\ 1, 2,3 Department of Oral and Maxillofacial Surgery, Saveetha Institute of \\ Medical and Technical Sciences Chennai, Tamilnadu, India.
}

\section{ABSTRACT}

\section{BACKGROUND}

Many studies have been published in the past on aerosols as a source of infection, despite their wide application in the field of dentistry. Most dental procedure and minor oral surgical procedures done with the use of surgical handpieces cause the formation of aerosol and splatter which are commonly contaminated with either bacteria, viruses, fungi, often with blood. With most of the procedures pertaining to oral surgery being related with the use of surgical handpiece, it results in maximum bacterial and viral load to both the patient and the operator. In this study we wanted to assess the microbiological load in various oral surgical procedures such as impactions, alveoloplasty, and other minor oral surgical procedures.

\section{METHODS}

The study population included 10 handpieces that are routinely used for minor oral surgical procedure in the Department of Oral and Maxillofacial Surgery at Saveetha Dental College, Chennai. The handpiece was selected randomly from 10 different operators, and samples were collected pre-operatively, and post operatively. They were subjected to microbiological assay and did a quantitative analysis of how much of CFU were present in $24 \mathrm{hrs}$. and $48 \mathrm{hrs}$. respectively.

\section{RESULTS}

10 handpieces were studied which were randomly selected, and two samples were taken both pre-operatively and post-operatively. Being a quantitative analysis study, only the microbial colony load was assessed at $24 \mathrm{hrs}$. and $48 \mathrm{hrs}$. whether there is any presence or absence of colony. Statistical analysis showed that there were significant differences between pre-operative and post-operative samples $(\mathrm{p}<0.05)$.

\section{CONCLUSION}

Bacterial count showed that disinfection in between procedures alone is not sufficient as back flow of the contaminants is noted. The design of the handpiece has to be modified to prevent any cross-infection.

\section{KEY WORDS}

Microbiological Assay, Bacterial Load, Handpiece, Cidex (Glutaraldehyde) Solution, Handpiece Design, Incubation
Corresponding Author: Dr. Swetha Murali.

New No. 24, Old No. 173, Rangarajapuram Main Road Kodambakkam Chennai600024, Tamilnadu, India.

E-mail: swetha6792@gmail.com

DOI: $10.14260 /$ jemds/2020/758

How to Cite This Article:

Murali S, Madhulaxmi M, Muthusekar MR, et al. To evaluate bacterial contamination of surgical handpiece used in minor oral surgical procedures in dentistry. J Evolution Med Dent Sci 2020;9(46):3463-3468, DOI: $10.14260 /$ jemds/2020/758

Submission 07-07-2020,

Peer Review 02-09-2020,

Acceptance 08-09-2020,

Published 16-11-2020.

Copyright (C) 2020 Swetha Murali et al. This is an open access article distributed under Creative Commons Attribution License [Attribution 4.0 International (CC BY 4.0)] 


\section{BACKGROUND}

A high-speed motorized surgical hand piece is used in several dental procedures, and most of the minor oral surgical procedures involve the usage of surgical hand piece, and the proper recommendation for safe decontamination and sterilization mainly consists of rinsing with water thoroughly, followed by washing with soap or any liquid detergent, and scrubbing the surface by manual wash, and finally subjecting it for sterilization by autoclave. ${ }^{1}$ however, many health care professionals and assistants tend to wipe the instrument surface with $70 \%$ weight / volume of alcohol without even cleaning the tool in between patients, such method of minimal surface cleaning is not sufficient. Studies have proven that only disinfecting the surface of high-speed hand piece with $70 \%$ weight / volume of alcohol found that the surface disinfectant method used in clinical practice are not mostly safe. ${ }^{1}$ The risk of infection due to insufficient sterilization of instruments between procedure and prior to procedure is one of the major reasons for hospital acquired diseases both to the patient and the operator. Most of the dental instruments used are potential sources of microbial infection among patients. They host and carry several microorganisms, which can result in nosocomial infections also known as hospital acquired infections. ${ }^{2-6}$

A high-speed hand piece (HSH) is used in several dental and minor oral surgical procedures, such as dental prophylaxis, preparing cavities for restoration, odontosections, osteotomies, impactions, alveoloplasty and other minor oral surgical procedures. ${ }^{7-8}$

This modern innovative gadget not only has benefits but has a wide range of application in the field of dentistry as well as oral surgery, but this advance modern technology comes with a price, it has high maintenance and different standard for safety and disinfection measures. The official recommendation by scientific societies for safe high-speed hand piece (HSH) decontamination is by rinsing with water, followed by washing with soap or detergent, and applying manual pressure to scrub off the surface contaminants, followed by autoclave sterilization. ${ }^{9-10}$

The use of $70 \%$ weight / volume of alcohol as a mode for disinfection process is not recommended as an only means of disinfection for critical and semi critical instruments, as it can only eliminate microorganisms with lower virulence, because there is no complete elimination of bacterial spores and prions.11-12 In spite of this reason, alcohol is still widely used as an intermediate surface-level disinfectant because it is cheaper than other surface disinfectants available in the market and is less toxic. Due to this it is considered to be nonspecific antimicrobial agent as it shows variations in its microbial disinfection action.

A surgical handpiece is an engine driven equipment used in surgical procedures such as extraction. Mainly in impaction, for alveoloplasty. The design of the handpiece is in such a way that there is backflow of the saliva and the irrigation solution used which predominantly contains microorganisms. The potential for contamination of the external surface of the handpiece and internal water and air lines has been welldocumented. ${ }^{13-14}$ Currently no study has demonstrated actual microbial contamination of high-speed air turbines used in a clinical environment. Also unknown are the effects of lubricating oils and cleaners on the microorganisms that might contaminate the internal components of the handpiece, or whether the air turbine is, in fact, rendered sterile by standard clinical autoclave procedures.

Sterilization of handpiece is a tedious process and a thorough cleaning of the internal and the external component of the handpiece is mandatory to avoid any cross-infection, or any nosocomial infection. To ensure a complete and efficient sterilization of handpiece and any other instrument, and to ensure that the steam can reach the whole surface that has to be sterilized, the instruments must previously be cleaned.15-18 Many studies also emphasized this point that it is essential that handpiece benefit from an optimal cleaning to ensure the effectiveness of their sterilization. ${ }^{19-25}$ On one side, the external cleaning of handpiece does not raise problems. On the other side, great difficulties remain in internal cleaning of the dental handpiece and parts where there can be entrapment of microorganisms due to back pressure flow of the coolant agent, these complex parts of the handpiece can pose to be the main culprit behind the contamination rate. ${ }^{26-28}$ Mainly because of the complex internal architecture of handpiece and the much reduced dimensions of the air / water pipes. This study thus analyses the microbial load in surgical handpiece.

\section{Study Design}

The study was conducted on 10 different surgical handpieces which were used by 10 different operators for different minor oral surgical procedures, each handpiece was given for sterilization prior to starting any procedures, and quantitative analysis was done pre-operatively, and post-operatively to assess the microbial load and the total colony count after 24 and 48 hours.

\section{Patients and Methods}

The present study is a quantitative clinical study designed to analyze the microbial load in handpiece used in minor oral surgical procedures. The study was conducted in line with the principles of the Helsinki Declaration; all surgical procedures were performed by 10 different operators for the same procedure in the department of oral and maxillofacial surgery.

\section{Study Population and Clinical Parameters}

The study included 10 handpieces which were randomly selected and there were 10 operators for the study and the procedure included impaction like minor surgical procedure. And the samples were collected in a sterile container and were labelled as clean- contaminated, that is sample size was taken immediately after autoclaving the handpiece, and contaminated sample that is the samples were collected postoperatively.

\section{METHODS}

This is a quantitative analysis study where in only microbial load and the total colony count was assessed at the end of 24 hrs. and $48 \mathrm{hrs}$. of incubation cycles.

The samples were collected in a sterile sample bottle and were labelled as pre-operative samples and post-operative sample, the sterile handpiece samples were taken by flushing the Cidex solution, this sample was labelled as clean sample, 
and the other sample was collected when the handpiece was autoclaved and was labelled as clean-contaminated, and the third sample was taken post procedure by flushing the handpiece with normal saline and the sample was labelled as contaminated.

All these samples were centrifuged at $3000 \mathrm{rpm}$ and then inoculated and later incubated in blood agar media for $24 \mathrm{hrs}$. and $48 \mathrm{hrs}$. at 37 degrees Celsius and the CFU was assessed at $25 \mathrm{hrs}$. and $48 \mathrm{hrs}$. post incubation.

All the samples that were collected were calculated using a colony counter and were tabulated separately as preoperative colony, clean- contaminated colony, and postoperative colony. At $24 \mathrm{hrs}$. and $48 \mathrm{hrs}$. after incubation.

Initially small colonies were formed but by end of $48 \mathrm{hrs}$. there was significant rise in colonies, and thus indicating that a better sterilization method has to be implemented so as to reduce any nosocomial infection.

\begin{tabular}{|cccc|}
\hline Handpiece & $\begin{array}{c}\text { Pre-Op } \\
\text { CFU / CCML }\end{array}$ & $\begin{array}{c}\text { Clean } \\
\text { Contaminated } \\
\text { CFU / CCML }\end{array}$ & $\begin{array}{c}\text { Post-Op } \\
\text { CFU / CCML }\end{array}$ \\
Handpiece 1 & 0 & 3 & 10 \\
Handpiece 2 & 0 & 2 & 18 \\
Handpiece 3 & 1 & 3 & 23 \\
Handpiece 4 & 0 & 3 & 15 \\
Handpiece 5 & 2 & 4 & 17 \\
Handpiece 6 & 1 & 5 & 37 \\
Handpiece 7 & 0 & 3 & 43 \\
Handpiece 8 & 2 & 3 & 23 \\
Handpiece 9 & 1 & 2 & 21 \\
Handpiece 10 & 1 & 2 & 12 \\
\hline Table 1. Total Colonies Formed at Various Stages of Sample Collection \\
\hline \multicolumn{4}{r}{}
\end{tabular}
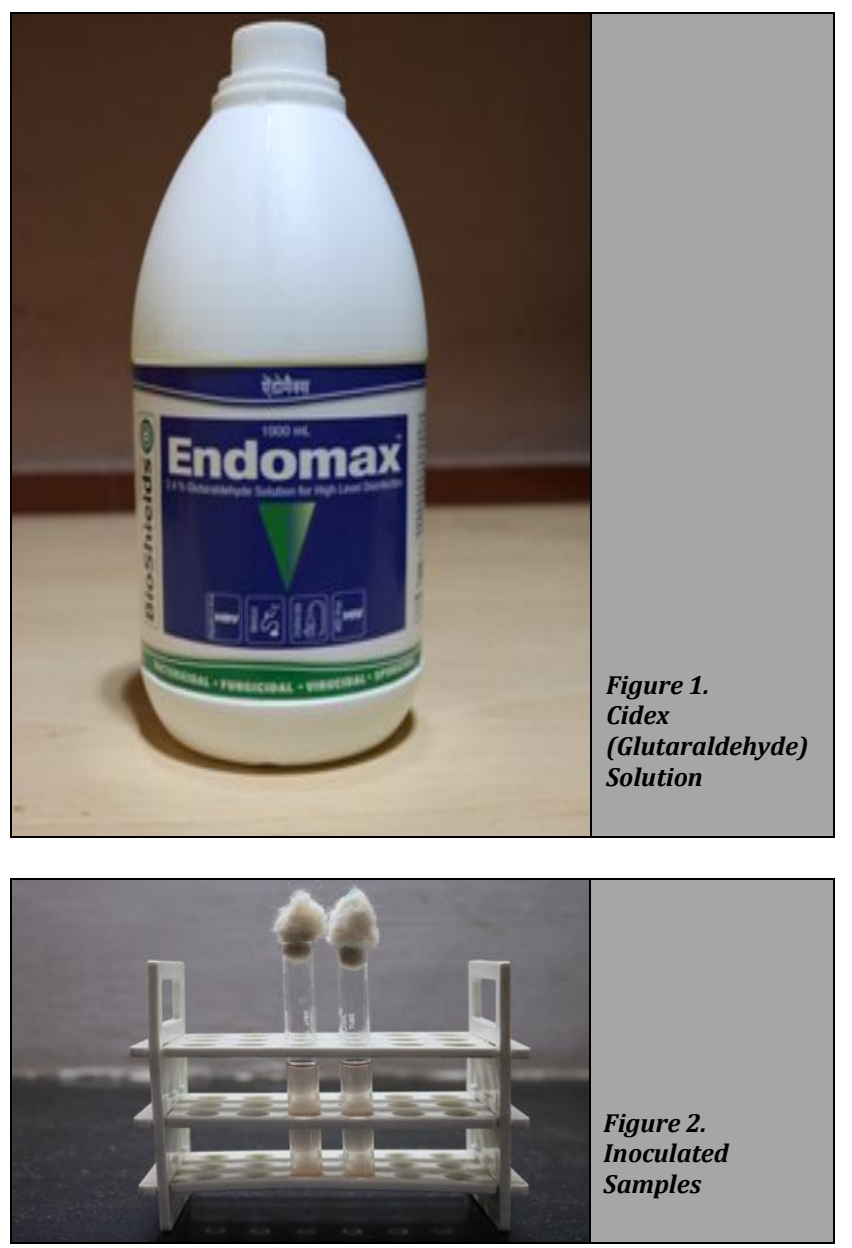
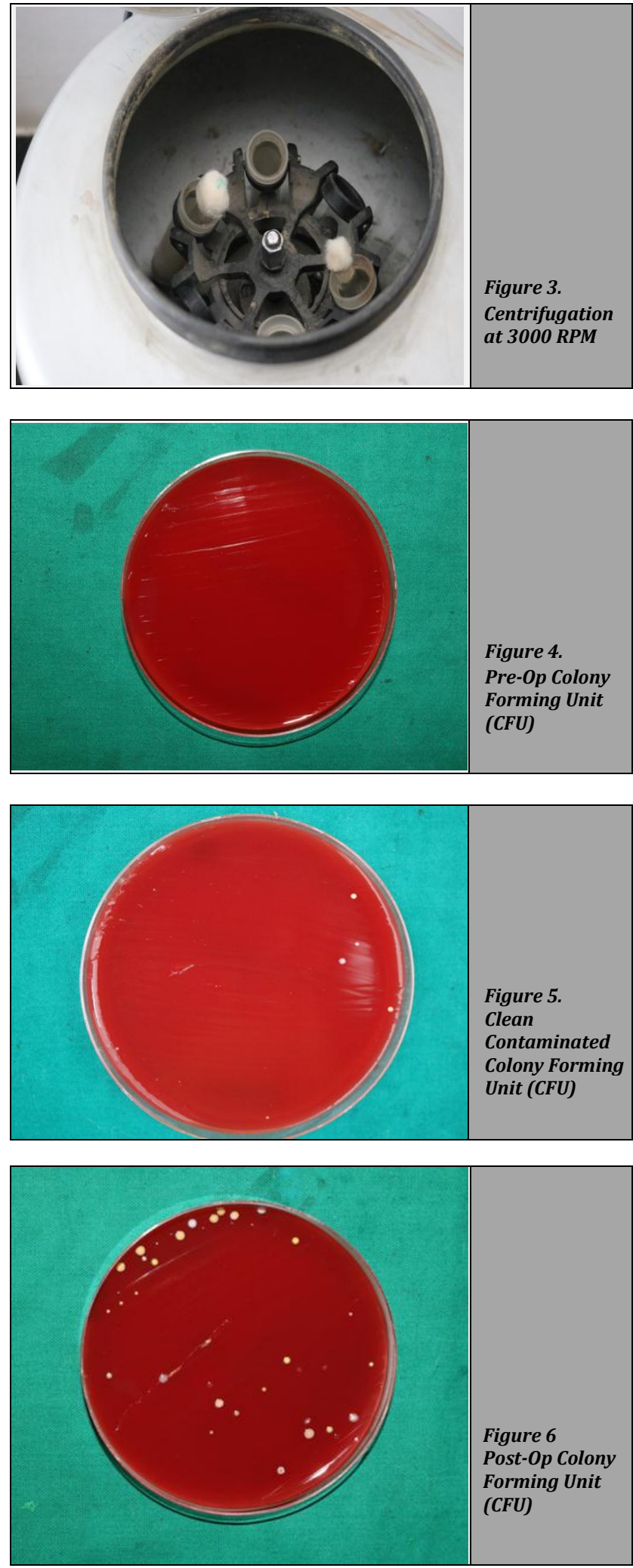

\section{Inclusion Criteria}

Only some minor surgical procedures like impaction were taken into consideration for the study.

\section{Exclusion Criteria}

1. No other surgical procedures were taken into sample.

2. No sterile handpiece was included in the study. 


\section{Sample Size Calculation}

$\mathrm{T}$ tests - Means: Difference between two dependent means (matched pairs)

Analysis: A priori: Compute required sample size

Input: Tail $(\mathrm{s})=$ One

Effect size $\mathrm{dz}=0.9128709$

$\alpha$ err prob $=0.05$

Power $(1-\beta$ err prob $)=0.8$

Output: Noncentrality parameter $\delta=2.738613$

Critical $\mathrm{t}=1.859548$

Df $=8$

Total sample size $=10$

Actual power $=0.802967$

A total of 10 handpieces were selected for the study and all the handpiece were sterile and autoclaved and the samples were collected both pre-operatively and post-operative before subjecting it for microbial analysis.

\section{RESULTS}

There was a significant increase in the microbial load after 24 hrs. of incubation in post-operative samples. The colony forming unit for each sample has been depicted in the bar chart given below.

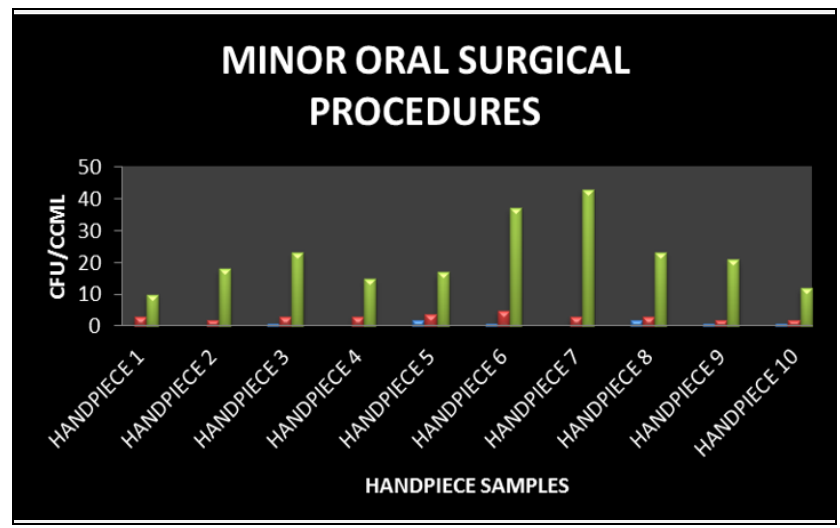

Table 2: Colony forming unit (CFU) at pre-operative sample, at clean contaminated and post-operative sample in 10 different handpieces, blue indicates pre-operative microbial load, red indicated clean contaminated microbial load, and green indicates post-operative microbial load.

According to the graph we could infer that the microbial load was more post-operatively in all the handpieces which were used for different minor oral surgical procedures, but there were presence of colonies in handpieces pre-operatively as well, this indicates that in spite of following CDC guidelines for sterilization for high critical equipment's and even cleaning the sterile equipment with glutaraldehyde prior starting any procedure we could find very few colonies in the handpiece, which indicates the risk of cross infection and contamination. Hence proving that a better sterilization protocol has to be devised with change in handpiece design as reverse flow of the coolant agent into the handpiece component is what is causing the presence of colonies in a sterile equipment stating that in spite of sterilization there is presence of microbial load, thus better sterilization protocols has to be implemented as well as modifications in surgical handpiece design is recommended
Pertaining to the field of oral surgery aerosol contamination is most common. Aerosols are mostly liquid and solid particles, with a particle size varying from $50 \mu \mathrm{m}$ or less in diameter and are mostly suspended in air. Aerosol is one of the primary culprit for cross contamination and infection in the field of dentistry and splatter is usually described as a mixture of air, water and / or some solid substances along with contaminants; whereas water droplets in splatter varies in size from $50 \mu \mathrm{m}$ to several millimeters in diameter and are not visible to the naked eye. Air-water aerosol produced during dental treatment procedures emerges from a patient's mouth and mixes with the surrounding air, thus resulting in this aerosol formation. Because air contained in this space is the air breathed by dentist and patient, its composition is what is mostly present around the dental operative unit and poses a potential threat to the patient as well as the dentist's health. Thus, it's important to follow the sterilization protocol to avoid any cross contamination and nosocomial infections.

\section{DISCUSSION}

We have been implementing time tested practices and slightly modernized them. The earliest form of dentistry involved treating teeth related problems with simple drills to prepare cavity and finish it with restoration, and most of these were craftsman and not professional dentists and the tools they used were not modern gadgets unlike what we have today with the help of modern science and technology. The reconstruction of this ancient form of dentistry and the ideas from the age old methods of practice showed that the methods used were reliable and effective Mostly cavities of $3.5 \mathrm{~mm}$ depth with concentric grooving and fissures will indicate the use of a drill tool but in ancient practices they used to follow more of atraumatic methods that is using simple hand driven drills which used to produce minimal to zero aerosols. Before the breakthrough of science and engineering in the pregizmotic times, mechanical hand drills were used. Like most hand drills invented at that time, the major flaw in the design was the speed, they were mostly quite slow, with speeds reaching only up to $15 \mathrm{rpm}$. In 1864, one of the breakthroughs in handpiece designs was done, British dentist George Fellows Harrington invented a clockwork dental drill named Erado, the device was much faster than earlier drills invented, but was comparatively very noisy. Likewise, in 1868, American dentist George F. Green came up with a pneumatic dental drill powered with pedal-operated bellows, and James B. Morrison devised a pedal-powered bur drill in 1871, these handpieces were quite efficient at that time but still wasn't efficient as compared to the modern-day handpieces. And today we have high speed motorized drills with variable speeds and power, the improvement and advancement of technology has also resulted in the increase rate due to cross contamination and nosocomial infections leading to several vulnerable diseases.

\section{Surgical Handpiece and Its Implications in Oral Surgery}

Dental handpieces have come a long way from the ancient times, in today's modification, , but even till now the engine 
drive and the internal components have not been modified adequately to restrain the back flow of the irrigation solution that's been routinely used and also the saliva that enters into the internal water coolant junction, all of these are high in bacterial load and thus can result in high chances of nosocomial infection.

In this current study samples were collected from the back end of the handpiece the junction between the handpiece and the water coolant point, the handpiece was first flushed with Cidex solution and that sample was collected in a sterile container and the next sample was collected post-operatively and the sample was collected by flushing normal saline and was collected in a sterile container.

In general practice, hydrogen peroxide is used as a disinfectant for sterilizing handpiece, but in cases where in the handpiece are used in between two procedures just a disinfection alone is not sufficient as there is still microbial load present in the coolant and the water line units, which has been proved by this quantitative microbiological assay. In this study glutaraldehyde has been used as a disinfectant solution, as glutaraldehyde has high anti-bacterial effect than hydrogen peroxide.

Glutaraldehyde is used in the field of biochemistry and also has various applications in disinfection processes, as it's an amine-reactive homobifunctional cross linker and fixative prior to SDS-PAGE, staining, or electron microscopy. The mechanism of action is it kills cells quickly by crosslinking their proteins and is usually it's used alone for disinfection or sometimes mixed with formaldehyde. As it's one of the first of two fixative processes to stabilize specimens such as bacteria, plant material, human cells and thus its most commonly used and preferred. A second fixative procedure uses osmium tetroxide to crosslink and stabilizes cell and organelle membrane lipids. Fixation is usually followed by dehydration of the tissue in ethanol or acetone, followed by embedding in an epoxy resin or acrylic resin, this method is quite cumbersome and time consuming.

In this study we did an assessment of the handpieces by doing a culture growth assessment prior to the procedure and also after the procedure, there was no complete absence of any microbial growth from the handpiece turbines which was a surprising finding in spite of following sterilization protocols. There are several possibilities to explain the failure to recover viable microorganisms, in clean handpiece which was taken out from the autoclaved pouches still had shown few microbial loads and after flushing the handpiece with Cidex solution there were presence of few microbial colonies and in certain samples there were no organisms found and post-operatively there was significant increase in the microbial colony. The only logical explanation to find the presence of microorganisms in a clean sterile handpiece was due to the back-pressure flow from the handpiece while doing surgical procedures which has resulted in a high possibility of contamination, in spite of following the necessary disinfection and sterilization protocol.

Effective and efficient infection control has become an important step to provide safe dental care to patients. To achieve efficient sterilization, various sterilization and disinfection methods are used in dentistry for the decontamination of various semi critical and critical dental instruments. Dental handpieces have become an indispensable part of everyday practice in clinical dentistry. Transmission of pathogens through the dental handpieces is one of the important potential routes for the spread of infection and causing nosocomial infections. There is a long history of infections transmitted through the dental handpieces that necessitates the need for proper decontamination of the dental surgical instruments prior to use in a patient. And the importance of inter patient sterilization is also important while performing multiple procedures in a single day, due to a buzzing practice in most dental units and institutions, many of them fail to disinfect the surgical handpiece instead they choose to just disinfect the surface by cleaning the surface thoroughly which might not be efficient in preventing any cross contamination.

It is an important aspect that dental practitioners should be made aware of the importance of following the proper steps in the autoclave sterilization procedure as recommended by the centers for disease control (CDC). Centers for disease control (CDC) states that the dental field requires straightforward, and consistent guidelines on the best and efficient way to reprocess and efficiently sterilize dental handpiece devices between different procedures and between different patients. Centers for disease control (CDC) guidelines for infection prevention and control states that, between patients, dental health care personnel (DHCP) should clean and heat-sterilize handpieces and other intraoral instruments that can be removed from the air and waterlines of dental units and all those units which come in direct contact with aerosols has to be disinfected thoroughly. The following were the conclusive protocol formulated by centers of disease control (CDC) for sterilization of surgical handpiece;

1. Firstly, clean and subject the handpieces for heat sterilization along with other intraoral instruments that comes in direct contact with the aerosol along with the suction and waterlines of dental units.

2. For handpieces that are attached as a separate system and does not get attached to the air or water line unit, follow the manufacturer's instructions for sterilization of these devices.

3. If a dental handpiece cannot be heat sterilized and cannot be subjected for autoclaving and does not have FDA clearance, do not such handpieces.

In this study we used clean and pre autoclaved surgical handpiece prior doing any oral surgical procedures, in unused surgical handpieces also we could find presence of few colonies, so in spite of chemical disinfection and thermal sterilization the surgical handpiece had few bacterial contamination, proves that in spite of following the CDC protocol for sterilization, and the manufactures instructions for disinfection of the surgical handpiece, we were unable to get a complete clean handpiece, which suggests that we have to modify the sterilization protocol and improve on design of the surgical handpiece so as to prevent any back flow of the coolant and water which is the main culprit for contamination, since these portion of the surgical handpiece cannot be subjected to continuous sterilization as it will result in quick wear and tear of the mechanical components of the handpiece, so a newer modification of the surgical handpiece has to be done so that efficient cleaning and disinfection can be done to avoid any bacterial contamination and to prevent any sought of infection.

There are several newer improved handpieces in the market yet none have been ergonomically designed in a way 
so that complete thermal sterilization and chemical disinfection can bring down the bacterial and viral load to a complete zero, and it's also important for the dental practitioners to minimize the repeated usage of the same handpiece during inter patient procedures and frequently changing sterilized handpiece is to be recommended in dental office with strict protocol to be followed according to the CDC recommended procedures for sterilization for highly critical equipment's such as the dental handpiece.

\section{CONCLUSIONS}

Handpiece design has to be modified so as to prevent any backflow of the contaminants through the water coolant and to prevent any nosocomial infection. The present study was a quantitative analysis to assess the microbial load in the handpiece. Further study has to be conducted to isolate the organisms. There was significant difference in the microbial colony both in pre-operative samples and in post-operative samples. Statistical analysis showed that there were significant differences between control and treatment groups with $(\mathrm{p}<0.05)$.

Further studies can be done by implementing newer sterilization protocols for disinfection of surgical handpieces.

Financial or Other Competing Interests: None.

The author thanks the following people who helped with the collection of the data and the preparation of the data for analysis: Dr. M Madhulaxmi, Dr. M R Muthusekhar.

\section{REFERENCES}

[1] Kohn WG, Collins A, Clveeland JL, et al. Guidelines for infection control in dental health-care settings- 2003. MMWR Recomm Rep 2003;52(RR-17):1-61.

[2] Pinto FMG, Bruna CQM, Camargo TC, et al. The practice of disinfection of high- speed handpieces with $70 \% \mathrm{w} / \mathrm{v}$ alcohol: an evaluation. Am J Infect Control 2017;45(1):e19-e22.

[3] Jorge, AOC. Princípios de biossegurança em Odontologia. Rev Biocienc 2002;8(1).

[4] Jakush J. Questions to follow tv shows. ADA News 1992:2.

[5] Runnells RR. Handpiece sterilization? (Editorial). Control 1992;7(6):1.

[6] Deng XH, Sun Z, Qiao H, et al. The possibility of hepatitis B virus transmission through dental handpieces. Zhonghua Yu Fang Yi Xue Za Zhi 2005;39(3):199-202.

[7] Larsen T, Andersen HK, Fiehn NE. Evaluation of a new device for sterilizing dental high-speed handpieces. Oral Surg Oral Med Oral Pathol Oral Radiol Endod 1997;84(5):513-6.

[8] Zhou X, Huang H, He X, et al. Effect of manual cleaning and machine cleaning for dental handpiece. Hua Xi Kou Qiang Yi Xue Za Zhi 2013;31(4):369-71.
[9] Smith GWG, Smith AJ, Creanor S, et al. Survey of the decontamination and maintenance of dental handpieces in general dental practice. Br Dent J 2009;207(4):160-1.

[10] Walker J, Kingston R, Chell K. Evaluation of washer disinfector for use in dental practices. The preliminary program for IADR General Session - poster 2010.

[11] Simonetti D'Arca AS, Petti S, Tomassini E, et al. A new device for the disinfection of handpieces and turbines. Minerva Stomatol 1995;44(7-8):369-75.

[12] Larsen T, Andersen HK, Fiehn NE. Evaluation of a new device for sterilizing dental high-speed handpieces. Oral Surg Oral Med Oral Pathol Oral Radiol Endod 1997;84(5):513-6.

[13] Lewis DL, Boe RK. Cross infection risks associated with current procedures for using high-speed dental handpieces. J Clin Microbiol 1992;30(2):401-6.

[14] Ricci ML, Fontana S, Pinci F, et al. Pneumonia associated with a dental unit waterline. Lancet 2012;379(9816): 684.

[15] Perrin D. Les instruments dynamiques. Clinic 1999;20(7).

[16] Thiveaud D. Du nettoyage à la stérilisation. Le lavage: l'étape essentielle [In French]. Hygienes 2:27-34.

[17] Arbeitskreis Instrumentenaufbereitung. Traitement correct des instruments de chirurgie [In French]. 8th edn. 2004. Web site. Www.a-k-i.org. Accessed January 2015.

[18] Weightman NC, Lines LD. Problems with the decontamination of dental handpieces and other intraoral dental equipment in hospitals. J Hosp Infect 2004;56(1):1-5.

[19] Zhou X, Huang H, He X, et al. Effect of manual cleaning and machine cleaning for dental handpieces. Hua Xi Kou Qiang Yi Xue Za Zhi 2013;31(4):369-71.

[20] Kudhail R. Can oil lubricated dental handpiece be sterilized? Part 2. Literature review. Dent Update 2013;40(8):630-2.

[21] Musso P. Le lavage des dispositifs médicaux en stérilisation dans les établissements de santé: évolution, aspects techniques et réglementaires. IRBM News 2007;28(4):24-31.

[22] Arbeitskreis Instrumentenaufbereitung. Traitement correct des instruments dans les cabinet's dentaires. 3rd edn. 2005.

[23] WHO. Guidelines for safe surgery 2009. Safe Surgery Saves Lives. World Health Organization 2009; p. 124.

[24] Coppa A, Bondioli L, Cucina A, et al. Palaeontology: early neolithic tradition of dentistry. Nature 2006;440(7085):755-6.

[25] Stone age man used dentist drill. BBC News 6 April 2006.

[26] MSNBC (2008). Dig uncovers ancient roots of dentistry

[27] BDA museum: collections: dental equipment: clockwork drill and dental engine. Br Dent Assoc 2013. Retrieved 9 September 2015.

[28] Karnovsky MJ. A formaldehyde-glutaraldehyde fixative of high osmolality for use in electron microscopy. J Cell Biol 1965;27(2):1A-149A. 